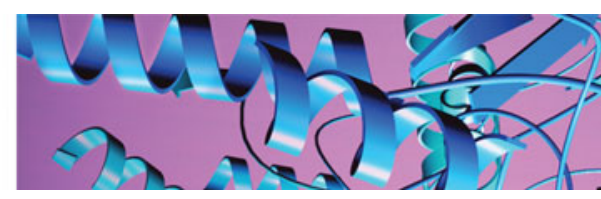

\title{
PERSPECTIVE
}

\section{Chemical materials and their regulation of the movement of molecules}

\author{
Robert Langer \\ MIT, Cambridge, MA, USA \\ Quarterly Reviews of Biophysics (2015), 48(4), pages 424-428 doi:10.1017/S0033583515000165
}

Abstract. Materials chemistry has been fundamental to the enormous field that encompasses the delivery of molecules both to desired sites and/or at desired rates and durations. The field encompasses the delivery of molecules including fertilizers, pesticides, herbicides, food ingredients, fragrances and biopharmaceuticals. A personal perspective is provided on our early work in this field that has enabled the controlled release of ionic substances and macromolecules. Also discussed are new paradigms in creating biomaterials for human use, the non-invasive delivery of molecules through the skin and lungs, the development of intelligent delivery systems and extensions to nanomedicine. With the advent of potentially newer biopharmaceutics such as siRNA, mRNA and gene editing approaches and their use being limited by delivery, future research in this field may be more critical than ever before.

Key words: Chemical materials molecular delivery.

Materials are used to control, and in many cases, direct the delivery of specific molecules. The applications are vast and include herbicides, pesticides, fertilizers, aquaculture, household products (e.g., fragrances), nutrition, and particularly drugs and biopharmaceuticals (Langer \& Wise, 1984). Hundreds of millions people use such products every year and annual sales are in the hundreds of billions of dollars (Taylor, 2011; Venugopal et al. 2009). Because of the potential impact of this field and the challenges of enabling new therapies (e.g. RNA, gene delivery), these numbers continue to grow rapidly.

My introduction to this field began 41 years ago and came through an unusual route. I began doing postdoctoral research with Dr. Judah Folkman at Harvard Medical School. We were attempting to isolate the first inhibitors of neovascularization, which were macromolecules. However, to do so required the development of a bioassay. None existed at that time. To create such a bioassay, we placed tumors in the corneas of rabbits and these tumors caused blood vessels to grow towards them over an 8-12

Authors for Correspondence: R. Langer, MIT, 500 Main Street, Room 76-661, Cambridge, MA, USA. Tel.: +1 617-253-3107; E-mail: rlanger@mit.edu weeks period. Using an ophthalmic microscope, we could measure the length of the longest vessel. However, we needed to place the macromolecular inhibitors in the corneas in such a way that did not cause any inflammation (or vascularization) in the cornea and that delivered these molecules continuously over this several month time period. Our thought was to embed the molecules and slowly release them from a tiny polymer system that could be placed in the cornea near the tumor (Fig. 1). Up until this time, only a few low molecule weight lipophilic molecules had been able to be continuously released from polymer vehicles and conventional wisdom suggested it was not possible to deliver other molecules. However, we made a serendipitous discovery (Langer \& Folkman, 1976). We found that by placing the molecules at a high enough density in certain polymers continuous release for many days could be achieved. This result was greeted with great skepticism by the polymer community which viewed the results as very surprising. For example, former Nature editor, Phil Ball, expressed this as follows 'It was widely believed at first that polymer delivery systems would not be equal to this task...But in 1976, Robert Langer and colleagues found that certain polymers, generally ones that were highly hydrophobic 


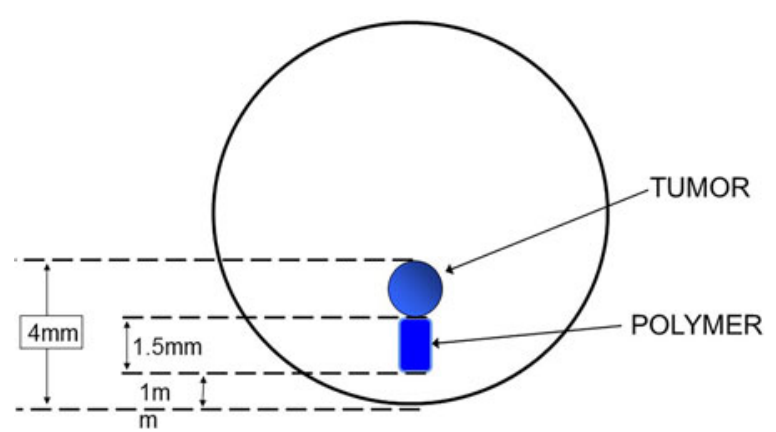

Fig. 1. Bioassay for studying tumor angiogenesis in the cornea.

(water-repellent) such as copolymers of ethylene and vinyl acetate, could be mixed with powdered proteins and formed into microspheres that would release the proteins at a steady, slow rate, persisting sometimes for up to one hundred days. There seemed to be no limit to the size of the large molecules that could be released controllably in this way, nor to their nature: proteins, nucleic acids, and polysaccharides (sugar polymers) could all be used.' (Ball, 1997) Similarly, Stannett et al. wrote 'Generally the agent to be released is a relatively small molecule with a molecular weight no larger than a few hundred. One would not expect that macromolecules, e.g., proteins, could be released by such a technique because of their extremely small permeation rates through polymers. However, Folkman and Langer have reported some surprising results that clearly demonstrate the opposite' (Stannett et al. 1979).

Without this discovery, the use of many such molecules would not be effective therapeutically since they are too large to be given orally or transdermally and they have half-lives of minutes or seconds when injected. Based on the above findings and related research by others, many new therapies became possible. For example, in 1989 a controlled-release system based in part on this work - microspheres made from a safe biocompatible copolymer of lactic and glycolic acid - was approved by the US Food and Drug Administration (FDA) for use with a large-molecule peptide drug that combats prostate cancer. This was the first polymeric controlled-release system for peptide-based drugs to find medical approval, and it now provides the most widely used treatment for advanced prostate cancer. Hundreds of thousands of patients each year are treated with these systems (Lupron Depot ${ }^{\oplus}, Z^{2}$ oladex $^{\oplus}$, or Decapepty $\left.1^{\circ}\right)$. Related systems are now used in the treatment of schizophrenia (Risperdal Consta ${ }^{\oplus}$ ), alcoholism and narcotic addiction $\left(\right.$ Vivitrol $\left.^{\circ}\right)$, diabetes $\left(\right.$ Bydureon $\left.^{\mathrm{TM}}\right)$, hemostasis (Floseal $^{\oplus}$, Surgiflo ${ }^{\oplus}$ ) and many other medical treatments. Millions of patients are being treated every year with such systems (Langer, 2013). These systems also permitted the isolation of the first angiogenesis inhibitors (Langer et al. 1976) and nearly all angiogenesis inhibitors and stimulators (Langer, 2013). It is estimated that 500 million patients will use these inhibitors (Carmeliet, 2005).
Much work has also been done on understanding how such materials can be used to control the release of molecules through polymeric systems. For example, controlling the pore structures in these polymeric materials and developing mathematical predictions as to how to control release rates in appropriately designed polymer systems have been critically important (Balazs et al. 1985; Saltzman \& Langer, 1989).

\section{Expanding our early findings and creating new biomaterials}

For almost the entire 20th century, biomaterials for clinical use were not designed as such, but were off-the-shelf materials that clinicians found useful in solving a problem. For example, dialysis tubing was originally made of cellulose acetate, a commodity plastic. The polymers used in vascular grafts, such as Dacron, were derived from clothes. The materials used for artificial hearts were based on commercial grade polyurethanes. In the case of breast implants, silicones and polyurethanes were used. While, these materials allowed medical issues to be addressed, they also introduced significant complications. For example, dialysis tubing can cause the activation of platelets and complement; Dacron-based vascular grafts can only be used if their diameter exceeds about $6 \mathrm{~mm}$, otherwise reactions occur at the blood-material interface; and blood-materials interactions can also cause clot formation in an artificial heart resulting in strokes or other complications. Breast implants have also led to major medical problems (Peppas \& Langer, 1994). In contrast, we asked what should be in an ideal biomaterial, from a chemistry, biology and engineering standpoint and then synthesized these materials. For example, in controlled release we and others proposed utilizing polymers that display surface erosion, a property which could protect unstable molecules from water-induced aggregation, as well as prevent large amounts of drug from being released all at once. To address this issue, we reasoned that the polymer should hydrolytically (as opposed to enzymatically) degrade because enzyme levels vary from person to person and over time due to the changing environment surrounding a polymer implant. To achieve hydrolytically induced surface erosion, we hypothesized that hydrophobic monomers connected by water-labile linkages were necessary and predicted the bond structures (anhydrides) to achieve this. We then selected nontoxic monomers and synthesized polyanhydrides from them (Langer, 2012).

Many drugs, when given systemically, are toxic at concentrations high enough to treat local diseases. We created technologies using these polymers that enabled long acting local chemotherapy. Specifically, we, in collaboration with neurosurgeon Henry Brem, used these polymers to create a new approach for localized long-acting chemotherapy for brain cancer. The polymer is combined with a 
chemotherapeutic drug and constructed into a wafer (Gliadel $^{\triangleright}$ in this case) which is placed directly over the tumor region during surgery (Brem \& Langer, 1996). This approach allows extremely high-sustained levels of chemotherapy directed to the tumor with virtually no systemic side-effects, significantly extending the life of patients. In 1996, the FDA approved this delivery system. It was the first time in over 20 years that the FDA approved a new treatment for brain cancer involving a system that delivers chemotherapy directly to the tumor. Based on this work, other cancer delivery systems that deliver drugs directly to the tumor are either approved (in the case of carcinomatous meningitis) or in clinical trials (in the case of ovarian and skin cancers). The concept of local delivery has been applied to polymer-coated stents nearly completely eliminating restenosis (Morice et al. 2002), one of the major problems in cardiovascular disease treatment affecting millions of patients each year.

Another approach to create biomaterials developed by David Lynn, now on the faculty of University of Wisconsin - Madison, and Dan Anderson, now on the MIT faculty, when they were postdoctoral fellows in our laboratory at MIT involves synthesizing libraries of polymers, for example, poly $\beta$-aminoesters, using methods that lend themselves to high-thoughtput parallel synthesis and screening approaches. We synthesized thousands of such polymers and developed screening assays to identify useful polymers based on DNA binding, solubility, and cell transfection (Lynn et al. 2001). In early studies, some of these polymers displayed higher transfection efficiencies in cells than standard non-viral vectors like lipofectamine and polyethylenimine. This approach is currently being extended to the synthesis and screening of thousands of polymers and this is accelerating the rate at which non-viral vectors are discovered for clinical applications. Parallel synthesis is also leading to a better understanding of structure/function relationships that could be applied to the design of other types of polymer - based vectors. Approaches like those discussed above are not only be useful for DNA delivery, but for new methods of gene therapy such as RNAi (RNA interference) as well. In particular with Dan Anderson, we have created libraries of thousands of lipids and they are being used as delivery systems for RNAi (Akinc et al. 2008; Dahlman et al. 2014).

Another exciting research area in the future is the development of smart drug delivery systems that would be able to detect chemical signals in the body and release drugs in response to such signals. For example, someday the release could simply be turned on by an external source, for example, by telemetry. To this end, we conceived of creating a silicon microchip containing a number of wells that would have normally inert metal covers over them. The drug could then be loaded into each well underneath the covers (Fig. 2). By simply applying an external, one-volt signal

\section{(a) Prototype device}

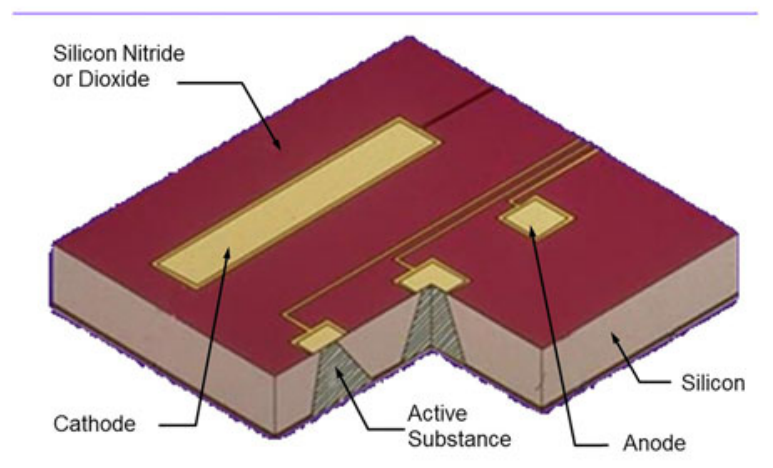

(b)

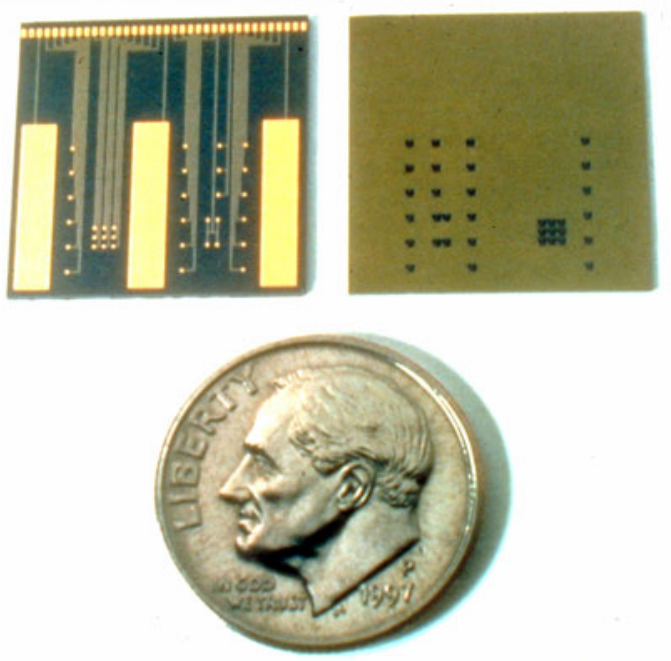

Fig. 2. Controlled release microchip. (a) Schematic device. (b) Top panel: top and bottom view of prototype chip with diffenent wells. Bottom panel: United States dime (to provide a reference for size).

selectively to any of these covers, the metal would dissolve (Santini et al. 1999) and the drug inside the well would come out. These are now in clinical trials for peptides such as parathyroid hormone (Farra et al. 2012).

\section{Extensions to non-invasive drug delivery}

Scientists have been exploring nearly every part of the body as a means of either delivering the drug to that body part or as a portal to the systemic circulation. These include the skin, the nose, the lungs, and the intestine. For example we have studied ultrasound as a means to enhance skin permeability. Joseph Kost, a former postdoctoral fellow and visiting scientist in my laboratories and now a Professor at Ben-Gurion University, discovered that ultrasound can increase the flux of substances through the skin (Levy et al. 1989) by temporarily disordering the lipid bilayers in the skin's outermost layer, the stratum corneum, which 
provides the principal barrier to drug diffusion. Using ultrasound, Samir Mitragotri, a former graduate student in our labs and my MIT colleague Dan Blankschtein and I showed that molecular fluxes to 5000 times normal can be achieved. Molecules the size of insulin or larger have been noninvasively transported through human skin models (Mitragotri et al. 1995). Glucose can also be detected this way by using ultrasound (Kost et al. 2000).

Lung delivery represents another important challenge. One of the greatest problems in lung delivery has been getting a sufficient number of aerosol particles into the lung so that inhaler devices could be reasonably small and the drug would not be wasted. Generally, less than $10 \%$ of the drug delivered from the inhaler is delivered to the lung by most conventional devices. In addition, repeated delivery every few hours is often necessary because lung macrophages clear most drugs rapidly. However, up until the mid-1990s scientists mostly focused on addressing this issue by designing improved aerosolizer devices and had paid little attention to the aerosol particles themselves. Then David Edwards, while a postdoctoral fellow in our lab at MIT (he is now a Professor at Harvard University) began to think of ways that one might design better aerosols. The concept that we conceived of was to design large highly porous aerosol particles with extremely low densities (Fig. 3). We considered that by lowering aerosol particle density, while increasing aerosol particle size, the aerodynamics of the aerosols would be altered in such a way that much larger particles might be able to enter the lung through an air stream with an extremely small and simple inhaler. We also reasoned that by increasing aerosol particle size while diminishing the mass density, the aerosol aggregation should be decreased. Due to their differences in surface area, the former are very hard to disaggregate whereas the latter have little propensity to stick together. In addition, aerosols are usually cleared relatively rapidly by lung macrophages. However, by making the aerosols larger, we thought that there would be decreased phagocytosis by the macrophages (Edwards et al. 1997). This approach is fundamentally changing how aerosols are being given to patients and is being used for the delivery of molecules like insulin for diabetes and dopamine in Parkinson's disease.

\section{Extensions to nanomedicine}

The original controlled-release materials we developed were small particles; in many cases these were microspheres. Clinically, these are injected subcutaneously or intramuscularly and the rate of drug release is controlled for a desired period of time. However, these systems cannot direct a drug to specific cells, other than by physical placement. To address this issue of targeting, nanoparticles are critical for delivering significant payloads of any drug into cells, particularly potential newer drugs like siRNA. However,

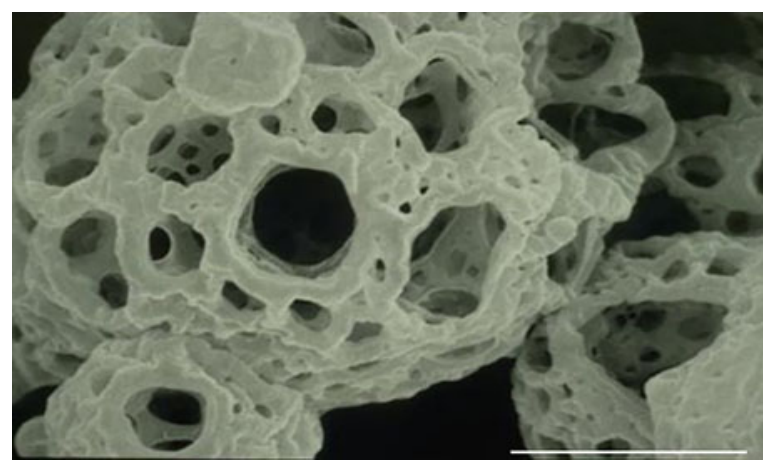

Fig. 3. Scanning electron microscope picture of large, highly porous aerosol particle.

polymeric nanoparticles injected into the body were destroyed almost immediately by macrophages, and were unstable because they aggregated. This made their use essentially nonexistent. In a 1994 paper (Gref et al. 1994), we addressed these problems. We discovered that nanoparticles composed of a block copolymer of polyethylene glycol and any other material such as poly lactic acid, and an added drug, could circulate for hours in vivo, be stable on the shelf for years, and not aggregate. These kinds of principles are now being widely used by many scientists and companies to practice 'nanomedicine' (Hrkach et al. 2012; Langer \& Weissleder, 2015; Service, 2010).

\section{Concluding remarks}

While enormous progress has been made in the creation of drug delivery systems, many challenges remain. One of the greatest challenges is to further enable drugs to be targeted to specific cells in the body perhaps by discovering new targeting molecules. One hope is that antibodies or appropriate sugars will be designed that might be complex with drug delivery systems to achieve this goal. Another potentially important area is the development of new vaccine delivery systems. Perhaps controlling the release of the vaccine in an optimal way would maximize the immune response. Alternatively, developing a desirable adjuvant and releasing it in an optimal manner could stimulate the immune system to produce desired antibody titers. A similar challenge is the development of new ways to deliver allergens that would avoid the current scheme of multiple injections. Such systems could be of enormous use in vaccinating people in the developing world where patients often cannot return for needed repeat injections. Another important area of research is delivery of drugs to not easily accessible sites such as the brain, nerves, the ear or sinuses.

There is little question that as time progresses, advances in materials based drug delivery systems will continue to be made and that someday drugs will be released precisely at the level they are required and to the exact places in the body where they are needed. Most importantly, newer and 
better therapies for patients will continue to evolve using these methods. Synthesizing and designing novel materials for controlling the movement of molecules is a remarkably exciting field. It enables one to create new principles in chemistry and materials science and to potentially use them to help millions, if not billions, of people worldwide.

\section{References}

Akinc, A., Zumbuehl, A., Goldberg, M., Leshchiner, E., Busini, V., Hossain, N., Bacallado, S., Nguyen, D., Fuller, J., Alvarez, R., Borodovsky, A., Borland, T., Constein, R., de Fougerolles, A., Dorkin, J., Jayaprakash, K., Jayaraman, M., John, M., Kotelianski, V., Manoharan, M., Nechev, L., Qin, J., Racie, T., Rattcheva, D., Rajeev, K., Sah, D., Soutschek, J., Toudjarska, I., Vornlocher, H. P., Zimmermann, T., Langer, R. \& Anderson, D. (2008). A combinatorial library of lipid-like materials for delivery of RNAi therapeutics. Nature Biotechnology 26, 561-569.

Balazs, A., Calef, D., Deutch, J., Siegel, R \& Langer, R. (1985). The role of polymer matrix structure and interparticle interactions in diffusion limited drug release. Biophysical Journal, 47, 97-104.

Ball, P (1997). Made to Measure: New Materials for the 21st Century, pp. 240-241. Princeton, NJ: Princeton University Press.

Brem, H. \& Langer, R. (1996). Polymer-based drug delivery to the brain. Scientific American: Science and Medicine 3, 52-61.

Carmeliet, P. (2005). Angiogenesis in life, disease and medicine. Nature 438, 932-936.

Dahlman, J., Barnes, C., Khan, O., Thiriot, A., Jhunjunwala, S., Shaw, T., Xing, Y., Sage, H., Sahay, G., Speciner, L., Bader, A., Bogorad, R., Yin, H., Racie, T., Dong, Y., Jiang, S., Seedorf, D., Dave, A., Sandu, K., Webber, M., Novobrantseva, T., Ruda, V., Lytton-Jean, A., Levins, C., Kalish, B., Mudge, D., Perez, M., Abezgauz, L., Dutta, P., Smith, L., Charisse, K., Kieran, M., Fitzgerald, K., Nahrendorf, M., Danino, D., Tuder, R., von Andrian, U., Akinc, A., Schroeder, A., Panigrahy, D., Kotelianski, V., Langer, R. \& Anderson, D. (2014). In vivo endothelial siRNA delivery using polymeric nanoparticles with low molecular weight. Nature Nanotechnology 9, 648-655.

Edwards, D., Hanes, J., Caponetti, G., Hrkach, J., Ben-Jebria, A, Eskew, M., Mintzes, J., Deaver, D., Lotan, N. \& Langer, R. (1997). Large porous aerosols for pulmonary drug delivery. Science 276, 1868-1871.

Farra, R., Sheppard, N., McCabe, L., Neer, R., Anderson, J., Santini, J., Cima, M. \& Langer, R. (2012). First in-human testing of a wirelessly controlled drug delivery microchip. Science Translational Medicine 4, 122ra21.

Gref, R., Minamitake, Y., Peracchia, M., Trubetskoy, V., Torchillin, V. \& LANGER, R. (1994). Biodegradable long-circulating polymeric nanospheres. Science 263, 1600-1603.

Hrkach, J., von Hoff, D., Ali, M., Andrianova, E., Auer, J., Campbell, T., De Witt, D., Figa, M., Figueiredo, M., Нorhota, A., Low, S., McDonnell, K., Peeke, E., Retnarajan, B., Sabnis, A., Schnipper, E., Song, J., Song, Y., Summan, J., Tompsett, D., Trojano, G., Van Geen Hoven, T., Wright, J., LoRusso, P., Kantoff, P., Bander, N., Sweeney, C., Farokhzad, O., Langer, R. \& ZALE, S. (2012). Preclinical development and clinical translation of a PSMA targeted docetaxel nanoparticle with a differentiated pharmacological profiles. Science Translational Medicine 4, $128 \mathrm{ra} 39$.

Kost, J., Mitragotri, S., Gabbay, R., Pishro, M. \& Langer, R. (2000). Transdermal monitoring of glucose and other analytes using ultrasound. Nature Medicine 6, 347-350.

LANGER, R. (2012). The struggles and dreams of a young chemical engineer. Chemical and Engineering News 90, 20-24.

LANGER, R. (2013). Biomaterials and biotechnology: From the discovery of the first angiogenesis inhibitors to the development of controlled drug delivery systems and the foundation of tissue engineering. Journal of Biomedical Materials Research 101A, 2449-2455.

Langer, R. \& Folkman, J. (1976). Polymers for the sustained release of proteins and other macromolecules. Nature 263, 797-800.

LANGER, R. \& WeISSLEDER, R. (2015). Scientific discovery and the future of medicine: nanotechnology. Journal of the American Medical Association 312, 135-136.

LANGER, R. \& WISE, D. (eds) (1984). Medical Applications of Controlled Release, Vol. I: Classes of Systems. Boca Raton, FL. CRC Press.

Langer, R., Brem, H., Falterman, K., Klein, M. \& Folkman, J. (1976). Isolation of a cartilage factor that inhibits tumor neovascularization. Science 193, 70-72.

Levy, D., Kost, J., Meshulam, Y. \& Langer, R. (1989). Effect of ultrasound on transdermal drug delivery to rats and guinea pigs. Journal of Clinical Investigation 83, 2074-2078.

LynN, D., AmIII, M. \& LANGER, R. (2001). pH-responsive polymer microsphere: rapid release of encapsulated material within the range of intracellular pH. Angewandte Chemie 40, 1707-1710.

Mitragotri, S., Blankschtein, D. \& Langer, R. (1995). Ultrasoundmediated transdermal protein delivery. Science 269, 850-853.

Morice, M. C., Serruys, P. W., Sousa, J. E., Fajadet, J., Hayashi, E. B., Perin, M., Colombo, A., Schuler, G., Barragan, P., Guagliumi, G., Molnar, F. \& Falotico, R. (2002). A randomized comparison of a sirolimus-eluting stent with a standard stent for coronary revascularization. New England Journal of Medicine 346, 17731780.

Peppas, N. \& Langer, R. (1994). New challenges in biomaterials. Science 263, 1715-1720.

Saltzman, W. \& LANGeR, R. (1989). Transport rates of proteins in porous materials with known microgeometry. Biophysical Journal 55, 163-171.

Santini, J., Cima, M. \& Langer, R. (1999). A controlled-release microchip. Nature 397, 335-338.

ServicE, R. (2010). Nanoparticle Trojan horses gallop from the lab into the clinic. Science 330, 314-315.

Stannett, V., Koros, W., Paul, D., Lonsdale, H. \& Baker, R. (1979). Recent advances in membrane science and technology. Advances in Polymer Science 32, 69-21.

TAYLOR, L. (2011). Drug delivery sales will hit $\$ 197 \mathrm{~B}$ in 2014, PharmaTimes Digital. http://www.pharmatimes.com/Article/1109-12/Drug_delivery_device_sales_will_hit_197B_in_2014.aspx.

Venugopal, J., Prabhakaran, M., Low, S., Choon, A., Deepika, G., Dev, V. \& Ramakrishna, S. (2009). Continuous nanostructures for the controlled release of drugs. Current Pharmaceutical Design 15, 1799-1808. 